\title{
Dietary supplementation with medium-chain TAG has long-lasting cognition-enhancing effects in aged dogs
}

\author{
Yuanlong Pan $^{1} *$, Brian Larson ${ }^{1}$, Joseph A. Araujo ${ }^{2,3}$, Winnie Lau ${ }^{2}$, Christina de Rivera ${ }^{2,3}$, Ruben Santana ${ }^{1}$, \\ Asa Gore ${ }^{1}$ and Norton W. Milgram ${ }^{2,3,4}$ \\ ${ }^{1}$ Nestlé Purina Research, One Checkerboard Square, 2RS, St Louis, MO 63164, USA \\ ${ }^{2}$ CanCog Technologies, Toronto, Canada \\ ${ }^{3}$ Department of Pharmacology, University of Toronto, Toronto, Canada \\ ${ }^{4}$ Department of Psychology, University of Toronto at Scarborough, Toronto, Canada
}

(Received 20 August 2009 - Revised 23 November 2009 - Accepted 4 January 2010 - First published online 9 February 2010)

\begin{abstract}
The present study focused on the hypothesis that dietary supplementation with medium-chain TAG (MCT) will improve cognitive function in aged dogs by providing the brain with energy in the form of ketones. Aged Beagle dogs were subjected to a baseline battery of cognitive tests, which were used to establish cognitively equivalent control or treatment groups. The dogs in the treatment group were maintained on a diet supplemented with $5.5 \%$ MCT. After an initial wash-in period, all the dogs were tested with a battery of cognitive test protocols, which assessed sequentially landmark discrimination learning ability, egocentric visuospatial function and attention. The groups were maintained on the diets for 8 months. The MCT-supplemented group showed significantly better performance in most of the test protocols than the control group. The group differences also varied as a function of task difficulty, with the more difficult task showing greater supplementation effects than the easier tasks. The group given the MCT supplement showed significantly elevated levels of $\beta$-hydroxybutyrate, a ketone body. These results indicate, first, that long-term supplementation with MCT can have cognition-improving effects, and second, that MCT supplementation increases circulating levels of ketones. The results support the hypothesis that brain function of aged dogs can be improved by MCT supplementation, which provides the brain with an alternative energy source.
\end{abstract}

Brain ageing: Cognitive functions: Dogs: Medium-chain TAG: Ketone bodies

Dog cognitive function, like that of other mammals, becomes impaired over the course of ageing, and it provides a model of human cognitive ageing ${ }^{(1,2)}$. Decline in energy metabolism is a common feature of ageing in animals, and it is one of the several processes that are closely associated with age-dependent cognitive decline. Rapoport et al. ${ }^{(3)}$ found that brain glucose metabolism was reduced by up to $30 \%$ between 3 and 12 months of age in rats. London et al. ${ }^{(4)}$ reported that brain glucose metabolism was significantly reduced in Beagle dogs at 6 years of age than in 1-year-old dogs. Further changes occurred later in life, but in a manner that varied between brain structures. Brain metabolic decline has also been reported in aged monkeys ${ }^{(5)}$ and human subjects ${ }^{(6)}$, and it appears to be particularly more pronounced in pathological ageing. Alexander et al. ${ }^{(7)}$ reported that cerebral glucose metabolism was significantly lower in old patients with Alzheimer's disease than in healthy old control subjects. Drzezga et al. ${ }^{(8)}$ traced the development of cognitive decline in patients with mild cognitive impairment, discovering that the clinical symptoms of Alzheimer's disease were associated with further declines in cerebral glucose metabolism. These data suggest that the age-associated reduction in cerebral glucose metabolism is a common feature in ageing, that the process involved may be progressive, starting around the middle age, and that metabolic decline contributes to cognitive decline associated with ageing.

One possible means of counteracting deficits in cerebral glucose metabolism is by nutritional supplementation. Although glucose metabolism is the primary source of brain energy, ketone metabolism provides an alternative pathway, which normally occurs under starvation conditions. Ketone bodies are a natural endogenous energy source mainly produced by the liver from mobilisation of endogenous body fat and utilised by extrahepatic tissues (brain, heart, kidney, muscle, etc.). Henderson ${ }^{(9)}$ has proposed that dietary supplementation with medium-chain TAG (MCT) can be used to increase levels of ketones in the brain. MCT are converted to ketone bodies by the liver and, to a lesser extent, by astrocytes in the brain. The ketone bodies could then be used by neurons as an alternative energy source to alleviate the deficit in glucose metabolism. To partially test this MCT supplementation hypothesis, Reger et al. ${ }^{(10)}$ provided an MCT supplement to patients with Alzheimer's disease and reported an improvement in cognitive function in a subset of subjects who were negative for the apo E $\varepsilon 4$ allele. They also found that cognitive improvement correlated positively with levels

Abbreviations: BHB, $\beta$-hydroxybutyrate; CBC, complete blood count; MCT, medium-chain TAG.

* Corresponding author: Dr Yuanlong Pan, fax +1 314982 5857, email yuanlong.pan@rdmo.nestle.com 
of $\beta$-hydroxybutyrate (BHB), a ketone body. More recently, Page et al. ${ }^{(11)}$ examined MCT injection in subjects with type 1 diabetes, and found that MCT were able to reverse impairment in an array of cognitive domains precipitated by hypoglycaemia. The present investigation explored the potential beneficial effects of MCT supplementation in a population of aged dogs. Following a programme of baseline cognitive assessment, the dogs were placed into groups that were fed either a diet containing $5.5 \%$ MCT or a control kibble diet. Over the course of 8 months on the two diets, the dogs were tested with a battery of cognitive test protocols that assessed learning ability, visuospatial function and attention. In the first test protocol, learning ability and visuospatial function were assessed using a landmark discrimination learning protocol. These tasks are intended to assess allocentric spatial ability, which entails utilisation of external landmarks to localise objects in space and is distinct from egocentric spatial ability, in which the subject uses its own body position to identify the location of external objects. We have previously found that performance in the landmark protocol is sensitive to age as well as to a variety of interventions, including maintenance on an antioxidant $\operatorname{diet}^{(12)}$ and treatment with a combination of lipoic acid and acetyl-L-carnitine ${ }^{(13)}$.

The second test protocol focused on egocentric spatial ability, and has been described by Christie et al. ${ }^{(14)}$. The protocol first examined the ability of the dog to selectively respond to an object based on the proximity of the object to its left or right side, and second, it examined the ability of the dog to reverse its original response. To assess attention, we used a modified protocol for studying oddity discrimination learning. We have previously described one version of this task, in which the dogs are trained to select the odd item out of three objects ${ }^{(15)}$. In the present investigation, we first trained the dogs to respond to one of two objects, and then examined the effect of adding additional distractor objects. The task can be used to assess selective attention because accuracy falls with an increase in the number of distractors.

\section{Materials and methods}

\section{Dogs and housing}

The present study used twenty-four Beagle dogs (ten males and fourteen females) of 7.5-11.6 (9.79 (SD 0.84)) years of age at the start of the study. The study protocol was approved by the CanCog Technologies Institutional Animal Care Committee, and it followed the guidelines of the Ontario Ministry of Agriculture. All the dogs had at least 6 months of previous cognitive test experience, which included training on a delayed-non-matching-to-position task and an oddity $\operatorname{task}^{(16)}$. Whenever possible, the dogs were group housed based on compatibility (up to four per pen) in 1.52 by $5.03 \mathrm{~m}$ pens. In some cases, the dogs were housed individually because of compatibility reasons. In such cases, the pens were divided. The dogs were also provided with environmental enrichment consisting of toys and beds, and the opportunity to play outside on a daily basis. Housing temperature and humidity were held relatively constant by automated temperature control and continuous ventilation. Room environmental conditions had design specifications as follows: single-pass air supply with 62.3 cubic metres filtered
Table 1. Ingredients and chemical composition of diets

\begin{tabular}{|c|c|c|}
\hline & Control & MCT \\
\hline \multicolumn{3}{|l|}{ Ingredients (\% as fed) } \\
\hline Plant protein* & 31.79 & 31.79 \\
\hline Animal protein $†$ & $20 \cdot 62$ & $20 \cdot 62$ \\
\hline Cereal grains $\ddagger$ & $30 \cdot 00$ & $30 \cdot 00$ \\
\hline Tallow & $12 \cdot 5$ & $7 \cdot 00$ \\
\hline MCT & 0.00 & $5 \cdot 50$ \\
\hline Vitamins and minerals & 3.24 & 3.24 \\
\hline Soyabean hulls & 1.40 & 1.40 \\
\hline L-Lys & 0.40 & 0.40 \\
\hline DL-Met & 0.05 & 0.05 \\
\hline \multicolumn{3}{|l|}{ Nutrient composition (\% as fed) } \\
\hline Moisture & 7.41 & $7 \cdot 10$ \\
\hline Ash & $6 \cdot 32$ & $6 \cdot 24$ \\
\hline Crude protein & $32 \cdot 80$ & $33 \cdot 10$ \\
\hline Crude fat & $18 \cdot 5$ & $18 \cdot 8$ \\
\hline Crude fibre & $2 \cdot 86$ & 2.47 \\
\hline Linoleic acid (\% of total fat) & $10 \cdot 1$ & $10 \cdot 2$ \\
\hline Caprylic acid (\% of total fat) & $<0.10$ & $24 \cdot 1$ \\
\hline Capric acid (\% of total fat) & $<0.01$ & 1.33 \\
\hline \multicolumn{3}{|l|}{ Energy content } \\
\hline Calculated ME $(\mathrm{kJ} / \mathrm{g}) \S$ & $17 \cdot 86$ & 18.06 \\
\hline
\end{tabular}

MCT, medium-chain TAG; ME, metabolisable energy.

* Plant protein was obtained from rapeseed, maize and soya.

$\dagger$ Animal protein was obtained from chicken and casein.

$\ddagger$ Cereal grains included maize, rice and wheat.

$\S$ Calculated based on the predictive equation for ME in dog foods ${ }^{(30)}$.

air changes per minute; relative humidity of $60 \pm 10 \%$; temperature of $21 \pm 3^{\circ} \mathrm{C}$; a natural light-dark cycle.

\section{Test diets}

The control diet was a commercial super premium-type product for adult dogs. The test diet was formulated by replacing $5.5 \%$ tallow with $5.5 \%$ MCT. The MCT preparation consisted of $97 \%$ caprylic acid and $3 \%$ capric acid. Both the diets were isoenergetic, manufactured by Nestlé Purina PetCare, Inc. (St Louis, MO, USA), and contained the same levels of protein, fat and carbohydrates. Dietary ingredient and chemical composition are provided in Table 1. Diet samples were sent to Nestlé Purina Analytical Laboratories (Nestlé Purina Petcare) for chemical analyses. Ash, crude fat, crude fibre, crude protein, moisture and fatty acid profile (linoleic acid, capric acid and caprylic acid) were measured based on the Association of Official Agricultural Chemists methods, and they were found to be 942.05, 922.06, 962.09, 990.03, $930 \cdot 15$ and $996 \cdot 06$, respectively.

The dogs were fed once daily for about an hour. The dogs were fed to meet their maintenance energy requirements estimated by the formula 'maintenance energy requirement $=460.6 \mathrm{~kJ} / \mathrm{d} \quad(110 \mathrm{kcal} / \mathrm{d}) \times$ body weight ${ }^{0.75,(17)}$. Dogs had free access to water via wall-mounted automatic system and/ or water bowls. The dogs were weighed weekly at the beginning of the study, and the food that was provided was adjusted in order to maintain relatively constant body weights.

\section{Cognitive testing apparatus}

The testing apparatus consisted of a wooden box that was approximately $0.609 \mathrm{~m} \times 1.15 \mathrm{~m} \times 1.08 \mathrm{~m}^{(18)}$ in size and was a modified version of the Wisconsin General Test Apparatus 
widely used in cognitive assessment of primates. The front contained three height-adjustable gates through which the dog responded. The experimenter was separated from the dog by a plastic partition containing a one-way mirror and a hinged door. The tray was made of Plexiglas and contained either one medial food well and two lateral food wells, or four equally spaced food wells, depending on the task. The food reward was the Pro Plan ${ }^{\circledR}$ Adult Wet Dog Food Chicken \& Rice Entrée (Nestlé Purina Petcare). Approximately, $1 \mathrm{~g}$ of the food constituted each reward, resulting in a maximum of $12 \mathrm{~g}$ of additional food, depending on the task.

\section{Baseline cognitive testing and randomisation}

During the baseline phase, all the subjects were tested with a variable delay version of the delayed-non-matching-to-position $\operatorname{task}^{(16)}$, a size discrimination learning task and a size discrimination reversal task ${ }^{(19,20)}$. Performance of the subjects in the three tests was ranked, and the ranking was used to place the subjects into two cognitively equivalent groups.

\section{Feeding and cognitive testing schedule}

One of the groups was then placed on a standard control diet and the other on a modified diet containing $5.5 \%$ MCT. The details about subject distribution and cognitive testing schedule are outlined in Table 2. After a 1-week wash-in, all the subjects were tested with a landmark protocol, which continued for up to $92 \mathrm{~d}$. At $100 \mathrm{~d}$ after the initiation of the study, all the dogs were tested with an egocentric protocol. The variable object oddity task protocol was started on day 190 and was continued for $35 \mathrm{~d}$ (Table 2). For all cognitive testings, the subjects were tested once daily on one task at a time. The task order was largely based on previous test protocols. Thus, we have used a similar task order in previous studies $^{(14,15)}$.

\section{Landmark discrimination protocol}

The landmark discrimination protocol included three separate tasks: land-0; land-1; land- $2^{(21)}$. This protocol started with the subjects being trained to approach one of two objects based on their proximity to an external landmark (land-0). The subjects were tested on successively more difficult versions of the same general problem. The first task, referred to as land-0, utilised a yellow peg $(2 \mathrm{~cm} \times 2 \mathrm{~cm} \times 9 \mathrm{~cm})$ and two identical white coasters. The yellow peg was attached to one of the coasters. In each trial, the experimenter placed the food reward in either the left or the right food well and positioned the landmark accordingly. In this and subsequent tasks, food that was inaccessible to dogs was placed at the bottom of the coaster associated with non-reward in order to prevent the dogs from responding based on olfactory cues. Subjects were able to obtain food reward if they displaced the coaster attached to the yellow peg. The dogs were subjected to ten trials per day, with an inter-trial interval of $30 \mathrm{~s}$. Testing was done once a day and $6 \mathrm{~d}$ per week. The door was raised, and the tray was moved approximately $25 \mathrm{~cm}$ away from the dog for a brief inspection interval to enable the subject to see the spatial arrangement on the tray. The tray was then presented to the dog, and the dog was allowed to respond within $60 \mathrm{~s}$.

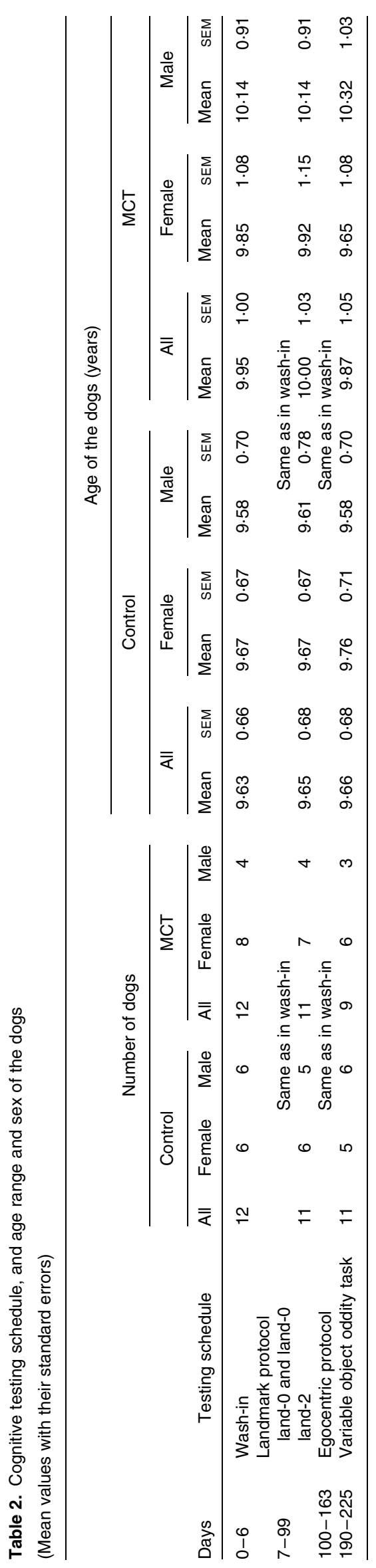


In this and all subsequent levels, the dogs were required to respond to the coaster closest to the landmark to obtain food reward. The correct side was determined randomly by computer, with the constraint that each side was correct in half of the trials of each test session. Each dog was allowed a maximum of thirty test sessions (300 trials) to learn to respond to the landmark-coaster combination used for land-0 task. A partial correction procedure was used in which the dogs were permitted to switch their response to the correct object after responding incorrectly to the coaster alone. Each animal was allowed only a single correction trial per session.

To move on from the first test to the second test (land-1), the dogs had to complete a two-stage criterion. The first stage was successfully completed when the subject responded correctly in at least nine of ten trials or in eight of ten trials over two consecutive days. The second stage was achieved when the subject responded in at least twenty-one trials (70\%) over three consecutive sessions. If a dog did not respond in a trial, it was assigned a score of 0.5 , which is the score that would have been obtained had the dog responded randomly. In this case, the dog was then given one extra day of testing to complete the thirty trials, and an average score of $70 \%$ over all the test days was required to pass the second stage.

For the land-1 task, the landmark was moved $1 \mathrm{~cm}$ medially and diagonally away from the edge of the coaster. Each dog was allowed a maximum of thirty test sessions (300 trials) to learn to respond to a stimulus associated with the landmark for land-1 task. A partial correction procedure was used in which the dogs were permitted to correct their response after making an error once in each session. Dogs that passed land- 1 task were then tested on land- 2 task, which was the same as land-1 task, except that the new landmark position was diagonally $1 \mathrm{~cm}$ away from the edge of the previous landmark position, and $2 \mathrm{~cm}$ from the edge of the coaster. Dogs underwent ten sessions in land-2 task.

\section{Egocentric protocol}

The egocentric protocol had three phases: a preference phase; an acquisition phase; a reversal learning phase. The egocentric acquisition protocol evaluates the animals' spatial learning ability to use a body-centred coordinate system to locate objects. The reversal protocol provides an additional measure of flexibility and executive function ${ }^{(14)}$. The preference phase occurred over a single test day and consisted of presenting the dog with ten discrete trials with identical objects covering both the lateral food wells and providing food reward on each trial. The side chosen most frequently was designated the preferred side, and it was assigned to be the positive side for the initial acquisition phase of testing. Thus, if the dog chose the object to its left most frequently, then the dog's left side was designated as its preferred side. For dogs that did not show a side preference (and responded five times to each side), a coin toss was used to determine the rewarded side.

For the acquisition (original learning) phase, the dogs were tested with twelve trials per session. Each trial consisted of a single presentation of the stimulus tray with a stimulus covering a reward on the preferred side lateral well or centre well. A second non-rewarded stimulus object covered a well towards the subject's non-preferred side. Consequently, the object closest to the dogs' preferred side was always rewarded. In any given trial, there were three possible spatial configurations (left-centre, left-right or right-centre). Each configuration occurred four times per test session. The original learning phase was successfully completed when the subject responded correctly in at least thirty-three of thirty-six trials over three consecutive test sessions.

All the dogs were subjected to two reversal tests (reversal 1 and reversal 2). The reversal phase was initiated on the day following the completion of initial learning. The test procedure was identical to that followed during the acquisition phase except that the rewarded position was switched to the opposite side. Thus, if the object closest to a dog's right was rewarded in acquisition testing, the object closest to its left was rewarded in reversal 1 testing. Reversal 1 testing was successfully completed when the subject responded correctly in at least twenty-six of thirty-six trials over three consecutive test sessions. Dogs that passed reversal 1 testing were then tested with reversal 2 testing, which was identical to reversal 1 testing, except that the rewarded position was switched to the opposite side of the reversal 1 testing.

A two-stage learning criterion was used. The first stage required subjects to perform either with at least $90 \%$ accuracy for $1 \mathrm{~d}$ or with at least $80 \%$ accuracy over two consecutive test days. The second stage required subjects to perform with above $70 \%$ accuracy over three consecutive days subsequent to passing the first stage criterion. The dogs had to pass the learning criteria in the first phase before they moved on to the next phase.

\section{Variable object oddity task}

This task was developed in order to assess attentional processes $^{(22)}$. The task had three phases: acquisition phase; 'same distractor' phase; 'different distractor' phase. The first phase examined acquisition of a two-choice discrimination problem that required the subjects to learn to selectively respond to a particular object to obtain a food reward. A two-stage learning criterion was used. The first stage required dogs to perform either with at least $90 \%$ accuracy for $1 \mathrm{~d}$ or with at least $80 \%$ accuracy over two consecutive test days. The second stage required dogs to perform with above $70 \%$ accuracy over three consecutive days subsequent to passing the first stage criterion. There were a maximum of nineteen sessions (one preference test and eighteen acquisition sessions). If a dog did not learn within eighteen sessions, it was given five sessions of remedial training and an additional ten sessions to reach the criterion. If a dog failed the discrimination, it was not tested further on this task.

Once a dog passed the acquisition phase, it was tested on the attention task. For the same and different distractor phases, each trial consisted of presenting to the dogs one, two, three or four objects, including the object that they had been trained to respond to during the acquisition phase. Thus, alternative objects served only as distractors, and the number of distractors varied from 0 to 3 . The same object was always the one associated with reward in the initial two-choice discrimination problem. The first seven sessions $(1-7)$ used the same procedure in which the negative stimuli used in discrimination served as the negative stimuli in the 
attention task. For sessions 8-14, the positive stimulus remained unchanged and a new distractor was used. In each test session, the subjects underwent three trials with zero distractors, three with one distractor, three with two distractors and three with three distractors. Both accuracy and speed of responding were used as dependent measures that were indicative of attentional processes.

\section{Body weight, food intake, clinical chemistry,} complete blood count and blood ketone bodies

Before the start of the study, baseline jugular blood samples were collected for measurements of BHB, CBC and clinical chemistry. These measurements were repeated after 4 and 8 months of treatment. Blood BHB samples were collected $2 \mathrm{~h}$ after feeding. Samples for clinical chemistry, $\mathrm{CBC}$ and $\mathrm{BHB}$ were sent to Advance Vet Lab (Mississauga, Ont., Canada) for analyses. Food intake was recorded daily, and body weight was recorded at 2-week intervals.

\section{Statistical analysis}

Errors were used as the dependent measure, and group comparisons were made using both Student's $t$ test and repeatedmeasures ANOVA. Values are means with their standard errors, except the cognitive data in the figures.

\section{Results \\ Baseline cognitive tests}

Fig. 1 shows that the performance of the two groups, control and MCT-treated dogs, did not differ in the baseline tests $(P>0 \cdot 05)$.

\section{Effect on performance in the landmark test}

The two groups were compared using a repeated measures ANOVA with total errors in land-0 and land- 1 tasks as withinsubject variables and group (supplementation $v$. control) as between-subject variable. The analysis revealed a significant effect of group $(P=0.015)$ and task $(P=0.0000001)$, and a significant group-by-task interaction $(P=0.043)$. The origin of these effects is shown in Fig. 2. The group effect reflects that the MCT group had fewer errors than the control group in both the tasks. The significant group-by-task interaction reflects that the treatment group differed from the control group in the land-1 task $(P=0 \cdot 02)$, but not in the land- 0 task $(P=0 \cdot 08)$.

Eleven dogs from each group completed the land-2 task. The groups were compared using a Student's $t$ test, which yielded a significant group effect $(t(2)=2 \cdot 242 ; P=0.0364)$. As illustrated in Fig. 3, the MCT-supplemented dogs performed at a higher level of accuracy than the controls (Fig. 3).

\section{Effect on performance in the egocentric test}

The data were analysed with repeated measures ANOVA, with task (original learning and first and second reversals) as within-subject variable and treatment as between-subject variable. The results revealed significant effects of group $(P=0.01)$ and task $(P<0.05)$. As illustrated in Fig. 4 , the
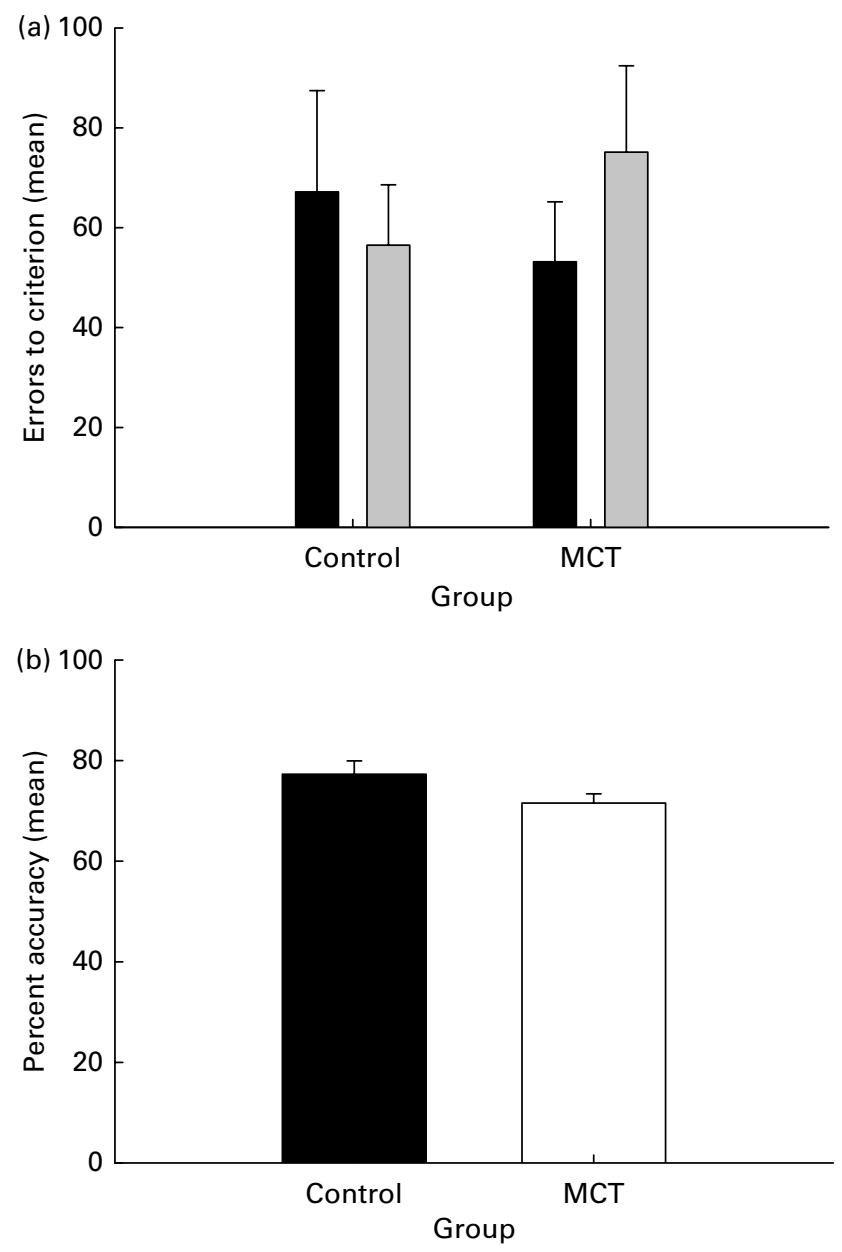

Fig. 1. Performance of dogs fed two diets in baseline cognitive tests. (a) Mean errors to criterion on the size discrimination and reversal learning tasks. (b) Percentage accuracy on the delayed-non-matching-to-position task. The data are means with their standard errors, $n$ 12. There were no statistically significant differences. MCT, medium-chain TAG; $\mathbf{~ , ~ s i z e ; ~}$ , size reversal.

task effect reflects greater errors during the reversal learning phase than in initial discrimination learning. In addition, more accurate learning was found in the second reversal task than in the first. The group effect reflects slower learning by the control group in all the three tasks (Fig. 4). Multiple comparisons were performed to analyse the group effect (Fisher's least significant difference) that revealed significant effects only in the second reversal learning task $(P=0.03)$.

\section{Effect on performance in the variable object oddity test}

In the acquisition phase of the attention task, the MCT group committed fewer errors than the control group, but the differences were NS $(P>0.05)$. The attention task was completed by ten control dogs and nine MCT dogs.

On the single object component, all the dogs performed at a very high level of accuracy, and the data from this component were not used in the statistical analysis. The data from 2, 3 and 4 distractor conditions were analysed by repeated measures ANOVA, with task (same $v$. different) and number of distractors (2, 3 or 4) as within-subject variables and treatment as a between-subject variable. 


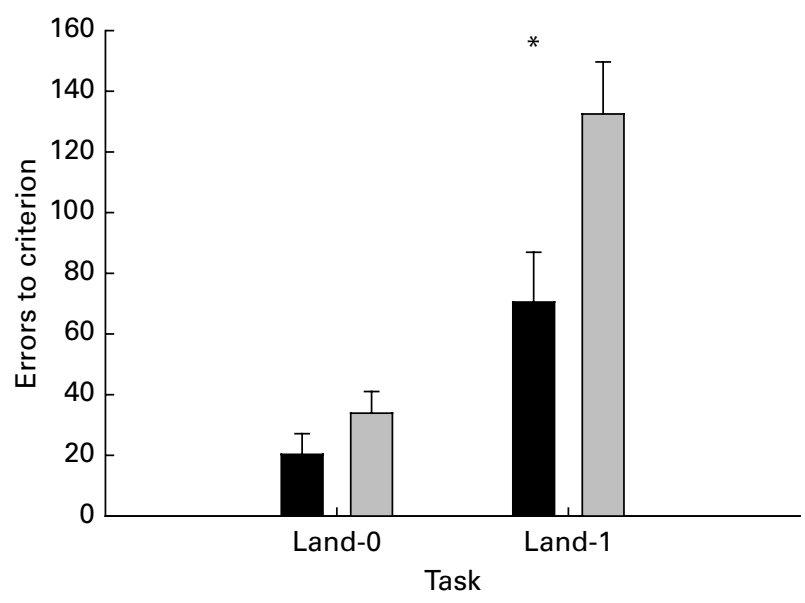

Fig. 2. Effects of dietary medium-chain TAG (MCT) supplementation on dogs' performance in object discrimination (land-0) and landmark discrimination (land-1) learning tasks. The performance was expressed as errors to criterion. The data are means with their standard errors, $n$ 12. * Mean values were significantly different $(P<0.05) \mathbf{\square}, \mathrm{MCT}$; 1 , control.

The results revealed significant effects of group $(P=0.018)$, task $(P=0.003)$ and number of distractors $(P<0.05)$. There were significant interactions between task and number of distractors $(P=0.03)$, and between group and number of distractors $(P=0.0016)$. These data are shown in Fig. 5, which illustrate that the dogs made fewer errors in the same task (Fig. 5(a), when the distractor was the negative object used in the original training) than in the different task (Fig. 5(b), when the negative object was originally unfamiliar). Fig. 5 also shows that the group-by-distractors interaction was a result of the control group performing more poorly than the treatment group when there were two or three distractors in both the same and different tasks. Performance was similar when the groups were only presented with one distractor. It was also noted that the group effect was larger in the different task (Fig. 5(b)) than in the same task (Fig. 5(a)).

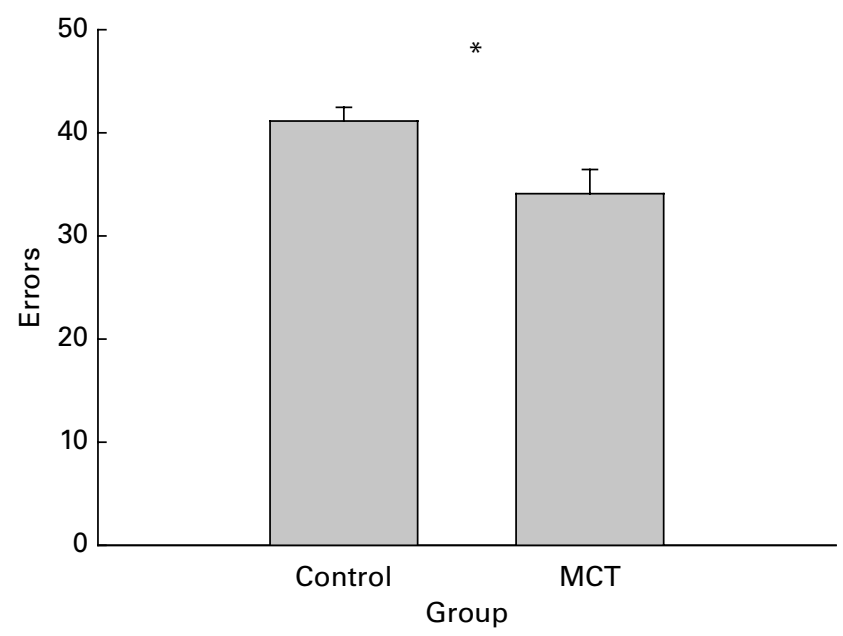

Fig. 3. Effects of dietary medium-chain TAG (MCT) supplementation on dogs' performance in complex landmark discrimination task (land-2). The data are means with their standard errors, $n$ 11. The performance was expressed as total number of errors over ten sessions. ${ }^{*}$ Mean values were significantly different $(P<0.05)$.

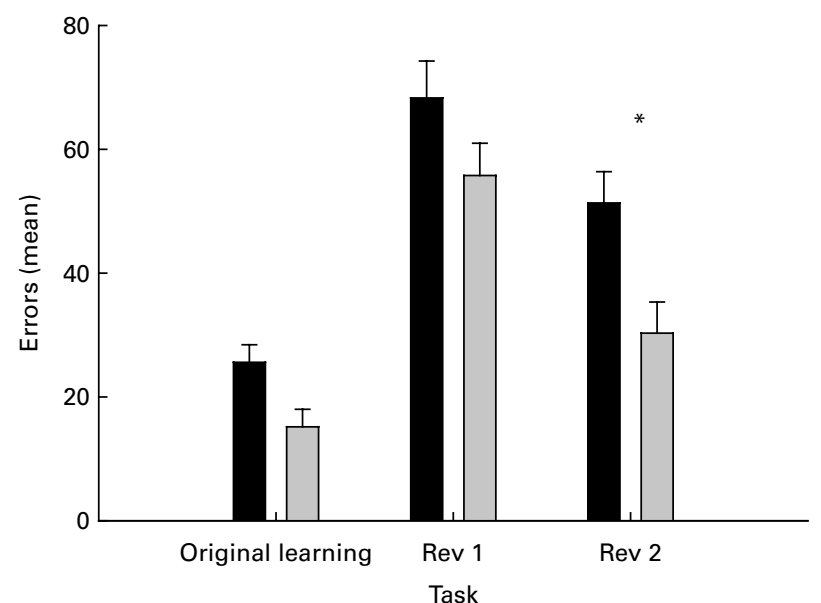

Fig. 4. Effects of dietary medium-chain TAG (MCT) supplementation on dogs' performance in the egocentric discrimination and two subsequent reversal learning tasks. The performance was expressed as errors to criterion. The data are means with their standard errors, $n$ 12. * Mean values were significantly different $(P<0.05)$. Rev 1 , reversal 1 ; Rev 2 , reversal 2; 口, Controls; 1 , MCT.

Effect on body weight, food intake and blood levels of $\beta$-hydroxybutyrate, complete blood count and clinical chemistry

Dogs in the MCT group consumed significantly $(P<0 \cdot 05)$ more food during the study, the average daily food intakes for control and MCT dogs were 144.45 (SEM 12.94) $g$ and $200 \cdot 32$ (SEM 12.94) g, respectively. Neither sex nor age significantly affected daily food intake within each group. Dogs in both the groups did not gain significant body weight (data not shown). Table 3 shows that the MCT diet significantly increased blood levels of BHB (which were taken under non-fasting conditions; $P<0 \cdot 05)$.

All the $\mathrm{CBC}$ parameters were within normal ranges for both the groups at baseline and throughout the study (data not shown). There were significant differences between control and MCT groups in lymphocytes (2.52 (SEM 0.26) $\times 10^{9}$ v. $1.76(\mathrm{SEM} 0.26) \times 10^{9} /$ litre $)$ and mean cell $\mathrm{Hb}$ concentration (347.00 (SEM 1.74) v. 340.00 (SEM 1.74) litre/ litre), both of which were higher in the treatment dogs than in the control dogs at baseline $(P<0.05)$. Monocytes $\left(0.26(\right.$ SEM 0.05$) \times 10^{9}$ v. $0.12($ SEM 0.05$) \times 10^{9} /$ litre $)$ and haematocrit $(0.45$ (SEM 0.01$)$ v. 0.41 (SEM 0.01 ) litre/litre) were significantly higher in MCT dogs than in the control dogs at 120 and $240 \mathrm{~d}$, respectively $(P<0 \cdot 05)$. Mean cell $\mathrm{Hb}$ concentration was significantly lower in the MCT dogs $(345.25$ (SEM 1.74) g/l) than in the control dogs $(355.58$ $($ SEM 1.74$) \mathrm{g} / \mathrm{l})$ at $120 \mathrm{~d}(P<0 \cdot 05)$. However, all the parameters of blood clinical chemistry analysis were within the normal ranges in both the control and the MCT dogs at baseline and throughout the study (data not shown), with few significant differences between the control and MCT dogs. The MCT dogs had significantly $(P<0.05)$ lower blood albumin (28.42 (SEM 0.75) v. 30.58 (SEM 0.75) g/l), creatinine $(47.00$ (SEM 4.35) v. 62.75 (SEM 4.35) $\mu \mathrm{mol} / \mathrm{l})$ and fasting blood glucose (3.08 (SEM 0.17) v. 5.43 (SEM 0.17) $\mathrm{mmol} / \mathrm{l})$, and significantly higher blood urea (7.26 (SEM 0.57) v. 5.60 (SEM $0.57) \mathrm{mmol} / \mathrm{l}$ ) than the control dogs at the baseline. Fasting blood glucose (4.39 (SEM 0.17) v. 4.88 (SEM 0.17) mmol/1) remained lower in the MCT dogs than in the control dogs at 

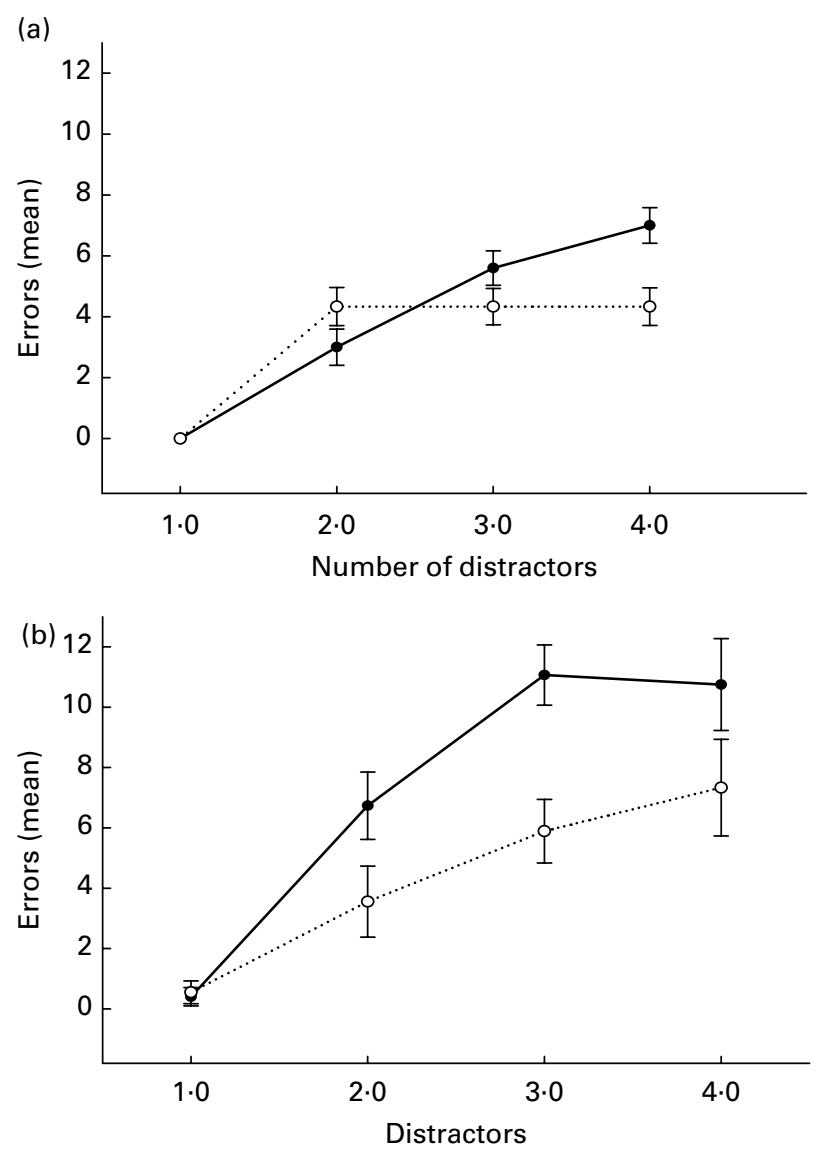

Fig. 5. Effects of dietary medium-chain TAG (MCT) supplementation on dogs' performance in two versions of the attention task. (a) Comparison of the performance of the control and MCT groups when subjects were tested with the same objects used in the initial training. The $Y$-axis shows errors, and the $X$-axis shows the number of distractors. (b) Comparison of the performance of the two groups when tested with the same rewarded object but with different non-rewarded objects. The performance was expressed as errors to criterion. The data are means with their standard errors, $n 9$ for the control group, $n 10$ for the MCT group. The statistical analysis revealed a significant interaction between number of distractors and treatments. $-\bullet-$, Control; $\cdot$. O.., MCT.

$120 \mathrm{~d}(P<0 \cdot 05)$. At $240 \mathrm{~d}$, serum alanine aminotransferase was higher in the MCT dogs $(102.83$ (SEM 15.71) U/l) than in the control dogs (58.17 (SEM 15.71) U/l; $P<0 \cdot 05)$. All thyroid parameters were within normal ranges in both the MCT-fed and control diet-fed dogs at baseline and throughout the study (data not shown).

Table 3. Effects of the medium-chain TAG (MCT) diet on blood $\beta$-hydroxybutyrate (BHB) after feeding in old dogs

(Mean values with their standard errors, $n 12$ )

\begin{tabular}{|c|c|c|c|c|c|c|}
\hline \multirow[t]{2}{*}{ Time (d) } & \multicolumn{2}{|c|}{0} & \multicolumn{2}{|c|}{120} & \multicolumn{2}{|c|}{240} \\
\hline & Mean & SEM & Mean & SEM & Mean & SEM \\
\hline \multicolumn{7}{|c|}{ Serum BHB $(\mu \mathrm{mol} / \mathrm{l})$} \\
\hline Control & $24 \cdot 25^{a}$ & 3.73 & $29 \cdot 83^{a}$ & $14 \cdot 17$ & $30 \cdot 67^{\mathrm{a}}$ & 14.77 \\
\hline MCT & $25 \cdot 08^{\mathrm{a}}$ & 3.73 & $123.83^{\mathrm{b}}$ & 14.17 & $110 \cdot 17^{b}$ & 14.77 \\
\hline
\end{tabular}

${ }^{a, b}$ Mean values within a row or column with unlike superscript letters were significantly different $(P<0.05)$.

\section{Discussion}

The primary focus of the present study was to test the hypothesis that dietary supplementation with MCT will have beneficial effects on cognitive function in aged dogs. A group of aged dogs were first subjected to a battery of cognitive tests to provide baseline data that were used to place the dogs in two cognitively equivalent groups. One group, the MCT treatment group, was then placed on a diet containing a food supplemented with $5.5 \%$ MCT, while the other group, control group, was fed an isoenergetic diet containing the same levels of fat, protein and carbohydrate. The results showed that the MCT-containing diet had positive effects on cognitive ability and increased serum ketone levels. Collectively, the results support the hypothesis that MCT supplementation can improve age-related cognitive decline by providing an alternative source of brain energy that can at least partly compensate for age-associated decrease in energy metabolism.

The first cognitive protocol consisted of three tasks: land-0, which assessed object discrimination learning, and land-1 and land-2, which assessed allocentric spatial ability and were more difficult tasks. Results indicated significant differences between the two groups overall, which were largely driven by differences in performance in land- 1 and land- 2 tasks and provided evidence that MCT supplementation improves visuospatial function in healthy old dogs.

Marginally significant treatment effects were seen in acquisition of the land- 0 task, which was concluded after the dogs had been on the treatment condition for about 2 weeks, and significant MCT effects were observed within a month during the feeding trial in acquisition of the land-1 task. This indicates that short-term administration of MCT supplementation is sufficient to improve learning ability.

The egocentric protocol commenced after approximately 3 months of maintenance on the MCT or control diets. This protocol had both an initial learning component, which required an egocentric learning strategy, and a reversal learning, which provided a measure of executive function and concept learning. There were significant group differences, which were largely driven by improved reversal learning in the MCT-treated groups. The greater group differences probably reflect differences in task difficulty.

The attention task had three phases. In the first phase, the groups were trained to discriminate between two objects, and group differences in learning were not found to be significant. In the second and third phases, the subjects were presented with the positive object and from 0 to 3 distractors. The results indicated that more distractors led to poorer performance, which is consistent with the task providing a measure of selective attention. Although the groups did not differ in initial learning, differences occurred in the second and third phases, suggesting that MCT supplementation improved the dogs' ability to focus their attention on the positive object.

Collectively, the cognitive assessment data showed that the performance of the MCT-supplemented group was superior to that of control group in the land-1 and land-2 tasks, and the egocentric learning and reversal task and on the variable object components of the attention protocol. By contrast, smaller and statistically insignificant group differences were noted in the land-0 task, the egocentric discrimination learning 
task and the object discrimination phase of the attention task. A primary difference between the tasks that showed significant treatment effects and those that did not is task difficulty: the more difficult tasks were the ones that showed the more significant effects. The observation that the effectiveness of the intervention varied with task difficulty is not surprising, and may represent a kind of floor effect. Moreover, we have previously shown a similar link between task difficulty and a cognition-modifying intervention. Beagle dogs fed an antioxidant-enriched diet showed improved learning of an oddity discrimination learning task only when the object similarity was increased to make the task more difficult ${ }^{(14)}$.

Serum levels of BHB at baseline and 120 and $240 \mathrm{~d}$ after initiation of the feeding trial were monitored to confirm the effectiveness of MCT supplementation to increase ketone levels in ageing dogs. Dogs fed the MCT diet had higher blood ketones, under fed conditions, confirming the ability of dietary MCT to increase blood ketone levels without a starvation regimen. The resulting ketone levels were well tolerated by dogs and were well below the ketone levels $(0.5 \mathrm{mmol} / \mathrm{l})$ induced by chronic starvation in $\operatorname{dog} \mathrm{s}^{(23)}$. All the parameters of CBC and blood biochemistry were within normal ranges at the end of the study in dogs fed either the MCT or control diet, indicating that the MCT diet had no adverse effects on the health of the dogs. The safety of dietary MCT in dogs was further confirmed by an independent safety study ${ }^{(24)}$, in which diets supplemented with $0,5,10$ and $15 \%$ MCT were fed to Beagle dogs for $90 \mathrm{~d}$. The results showed no signs of toxic effects in dogs at any MCT dose level.

There is a tight coupling between neuronal activity and cerebral glucose utilisation, and sustaining increased neuronal activity usually depends on increased ATP production ${ }^{(25)}$.

Normally, most of the ATP production in the neurons comes from glucose metabolism ${ }^{(25)}$. With reduced ability to metabolise blood glucose, old animals are not able to increase ATP production high enough to support increased neuronal activity, which may, at least partially, contribute to the decline in cognitive function in old animals. In addition, recent evidence also suggests a link between metabolic disorders and cognitive decline ${ }^{(26)}$. Since reduction in cerebral glucose metabolism is also reported in old human subjects ${ }^{(6)}$, it is highly likely that dietary MCT supplementation may be able to improve brain function in old people with and without any dementia symptoms.

There are also other possible explanations for the positive effects of MCT supplementation. Loss of PUFA involved in maintaining neural structure is another consequence of ageing ${ }^{(27)}$. Beyond serving as an alternative energy source for the brain, the cognition-enhancing effects of MCT may be related to brain distribution and concentration of PUFA ${ }^{(28)}$, which are involved in maintaining neural structure and known to decrease during ageing ${ }^{(27)}$. This suggestion is supported by a recent article that revealed increased levels of $n-3$ fatty acids in the brain in dogs treated with a programme of $\mathrm{MCT}^{(29)}$.

In summary, the present study shows that supplementation of MCT in ageing dogs can significantly increase blood ketone body concentrations under fed conditions and improve age-related decline in cognitive function by providing an alternative source of brain energy in old healthy dogs. The MCT-containing diet had no adverse effects on CBC and blood chemistry.

\section{Acknowledgements}

The authors confirm that they have no conflicts of interests associated with the present study. The study was entirely funded by Nestlé Purina Research. Y. P., B. L., J. A. A., C. d. R. and N. W. M. designed the present study, interpreted the results and prepared the manuscript. W. L. organised and supported the feeding trial. A. G. and R. S. contributed to the formulation and production of the diets, respectively. The authors wish to thank Wendell Kerr for his statistical analysis of the body weight, food intake and blood chemical data.

\section{References}

1. Adams B, Chan A, Callahan H, et al. (2000) The canine as a model of aging and dementia: recent developments. Prog Neuro-Psychopharmacol Biol Psychiatry 5, 675-692.

2. Tapp PD \& Siwak CT (2006) The canine model of human brain aging: cognition, behavior and neuropathology. In Handbook of Models for Human Aging, pp. 415-434. Burlington, MA: Academic Press.

3. Rapoport SI, London ED \& Takei H (1982) Brain metabolism and blood flow during development and aging of the Fischer344 rat. Exp Brain Res Suppl 5, 86-101.

4. London ED, Ohata M, Takei H, et al. (1983) Regional cerebral metabolic rate for glucose in beagle dogs of different ages. Neurobiol Aging 4, 121-126.

5. Noda A, Ohba H, Kakiuchi T, et al. (2002) Age-related changes in cerebral blood flow and glucose metabolism in conscious rhesus monkeys. Brain Res 936, 76-81.

6. Bentourkia M, Bol A, Ivanoiu A, et al. (2000) Comparison of regional cerebral blood flow and glucose metabolism in the normal brain: effect of aging. J Neurol Sci 181, 19-28.

7. Alexander GE, Chen K, Pietrini P, et al. (2002) Longitudinal PET evaluation of cerebral metabolic decline in dementia: a potential outcome measure in Alzheimer's disease treatment studies. Am J Psychiatry 159, 738-745.

8. Drzezga A, Lautenschlager N, Siebner H, et al. (2003) Cerebral metabolic changes accompanying conversion of mild cognitive impairment into Alzheimer's disease: a PET follow-up study. Eur J Nucl Med Mol Imaging 30, 1104-1113.

9. Henderson ST (2004) High carbohydrate diets and Alzheimer's disease. Med Hypotheses 62, 689-700.

10. Reger MA, Henderson ST, Hale C, et al. (2004) Effects of $\beta$-hydroxybutyrate on cognition in memory-impaired adults. Neurobiol Aging 25, 311-314.

11. Page KA, Williamson A, Yu N, et al. (2009) Medium-chain fatty acids improve cognitive function in intensively treated type 1 diabetic patients and support in vitro synaptic transmission during acute hypoglycemia. Diabetes 58, 1237-1244.

12. Milgram NW, Head E, Muggenburg B, et al. (2002) Landmark discrimination learning in the dog: effects of age, an antioxidant fortified diet, and cognitive strategy. Neurosci Biobehav Rev 26, 679-695.

13. Milgram NW, Araujo JA, Hagen TM, et al. (2007) Acetyl-Lcarnitine and alpha-lipoic acid supplementation of aged beagle dogs improves learning in two landmark discrimination tests. J FASEB 13, 3756-3762.

14. Christie LA, Studzinski CM, Araujo JA, et al. (2005) A comparison of egocentric and allocentric age-dependent spatial learning in the beagle dog. Prog Neuropsychopharmacol Biol Psychiatry 29, 361-369.

15. Milgram NW, Zicker SC, Head E, et al. (2002) Dietary enrichment counteracts age-associated cognitive dysfunction in canines. Neurobiol Aging 23, 737-745. 
16. Chan ADF, Nippak P, Murphey H, et al. (2002) Visuospatial impairments in aged canines: the role of cognitive-behavioral flexibility. Behav Neurosci 116, 443-454.

17. Laflamme DP (2007) Five minute veterinary consult: canine and feline. In Obesity, pp. 982-983. Ames, IA: Blackwell Publishing.

18. Milgram NW, Head E, Weiner E, et al. (1994) Cognitive functions and aging in the dog: acquisition of non spatial visual tasks. Behav Neurosci 108, 57-68.

19. Head E, Callahan H, Cummings BJ, et al. (1998) Visual-discrimination learning ability and beta-amyloid accumulation in the dog. Neurobiol Aging 19, 415-425.

20. Tapp PD, Siwak CT, Estrada J, et al. (2003) Size and reversal learning in the beagle dog as a measure of executive function and inhibitory control in aging. Learn Mem 10, 64-73.

21. Milgram NW, Adams B, Callahan H, et al. (1999) Landmark discrimination learning in the dog. Learn Mem 6, 54-61.

22. Christie LA, Araujo JA, de Rivera C, et al. (2010) Novel task for assessing attention in canines: age effects on accuracy and response latency. Neuroscience (In the press).

23. de Bruijne JJ, Altszuler N, Hampshire J, et al. (1981) Fat mobilization and plasma hormone levels in fasted dogs. Metabolism 30, 190-194.
24. Matulka RA, Thompson DV \& Burdock GA (2009) Lack of toxicity by medium chain triglycerides (MCT) in canines during a 90-day feeding study. Food Chem Toxicol 47, $35-39$.

25. Magistretti PJ (2009) Role of glutamate in neuron-glia metabolic coupling. Am J Clin Nutr 90, 875S-880S

26. Kodl CT \& Seaquist ER (2008) Cognitive dysfunction and diabetes mellitus. Endocr Rev 29, 494-511.

27. Freemantle E, Vandal M, Tremblay-Mercier J, et al. (2006) Omega-3 fatty acids, energy substrates, and brain function during aging. Prostaglandins Leukot Essent Fatty Acids 75, 213-220.

28. Taha AY, Ryan MA \& Cunnane SC (2005) Despite transient ketosis, the classic high-fat ketogenic diet induces marked changes in fatty acid metabolism in rats. Metab Clin Exp 54, $1127-1132$.

29. Taha AY, Henderson ST \& Burnham WM (2009) Dietary enrichment with medium chain triglycerides (AC-1203) elevates polyunsaturated fatty acids in the parietal cortex of aged dogs: implications for treating age-related cognitive decline. Neurochem Res 34, 1619-1625.

30. National Research Council (2006) Nutrient Requirements of Dogs and Cats. Washington, DC: National Academy Press. 